\title{
Le téléréglage niveau-puissance des groupes hydroélectriques au fil de l'eau
}

\author{
Remote power-water level control \\ for run-of-the-river power plant
}

PAR

JACQUES CHENAIS,

ANCIEN GLLEVE DE L'ÉCOLE POLXTECHNIQUE, INGÉNIEUR E.S.E., DIRECTEUR DE LA DIVISION MATERIEL NORMAL DES E ETARLISSEMENTS YEYRPIC

JEAN COHARD,

INGÉNIEUR E.I.H.

CHEF DU DÉPARTEMENT MESURES DES ÉTABLISSEMENTS NEYRPIC
ET

\begin{abstract}
Le réglage automatique de la charge des centrales hydro-électriques travaillant au fil de l'eau est en général compliqué par la présence d'oscillations de masse. Les appareillages qui assurent ce réglage automatique doivent introduire dans la boucle d'asservissement des éléments de stabilisation dont la valeur numérique varie dans chaque cas particulier. C'est pourquoi les deux régulateurs présentés sont conçus pour adapter avec facilité les paramètres de stabilité aux caractéristiques de l'installation.

Le premier type d'appareil réalise d'une manière très classique une correspondance de position entre le niveau réglé et l'ouverture de vannage. C'est donc un simple régulateur $\dot{a}$ "statisme permanent".

Le deuxième type d'appareillage, plus complexe, introduit dans la boucle de réglage à la fois un statisme permanent et un statisme temporaire; il autorise, de ce fait, des performances intéressantes dans des cas difficiles de régulation.
\end{abstract}

\begin{abstract}
Automatic head control for run-of-the-river power plant is usually complicated by the presence of mass oscillations. This automatic governing equipment must bring into play in the feedback circuit stabilising elements with numerical values which vary in each particular case. This explains why the two governors in question are designed to allow the stability parameters to be easily adapted to the characteristics of the installation.

The first system is a straightforward "permanent speed droop" governor, based on the conventional principle of linking level regulation to gate opening.

The second type is more complex and introduces both temporary and permanent speed droop into the feedback circuit, thus allowing very good results to be achieved in case where regulation is difficult.
\end{abstract}




\section{I. - INTRODUCTION}

Les répartitions de la charge entre les centrales thermo et hydroélectriques interconnectées ont fait l'objet de nombreuses et savantes études, faisant ressortir la complexité des facteurs divers : économiques, techniques, historiques même, qui conduisent, suivant les pays, suivant les situations géographiques, suivant le type des centrales et leurs servitudes propres d'exploitation, à des solutions très diverses.

En règle générale, restent complètement en dehors des systèmes de répartition de la charge d'un grand réseau, les centrales hydrauliques au fil de l'eau, trop étroitement asservies au débit qui passe, pour répondre à aucun autre critère.

C'est précisément sur ces centrales que nous désirons attirer l'attention, leur réglage convenable (c'est-à-dire assurant la meilleure production) pouvant être réalisé de façon automatique, grâce à des appareillages relativement simples et rustiques. On peut ainsi réduire, dans de nombreux cas, les charges d'exploitation, ces centrales étant souvent munies d'un appareillage automatique de démarrage, de sécurité et d'arrêt. Le réglage optimal de l'ouverture de leurs turbines est alors confié à un système automatique, plus simple et moins coûteux que l'ensemble de téléindications de niveau et de télécommandes qui permettrait de centraliser, dans un poste de dispatching régional, la surveillance de la charge et des niveaux de plusieurs centrales.

En théorie, le réglage d'une centrale hydraulique au fil de l'eau est très simple : maintenir, quel que soit le débit entrant dans la prise d'eau, Je niveau amont au maximum : il est en effet préférable de perdre une faible quantité d'eau par déversement occasionnel de la prise d'eau, que de laisser diminuer la chute, l'énergie du torrent se dissipant alors en remous dans les galets de son lit, à l'endroit où il devrait être en permanence recouvert par l'eau calme de la retenue.

En fait, le problème est souvent plus compliqué, du fait que nombre de retenues de prise d'eau n'ont qu'une surface très faible (retenues intermédiaires entre deux centrales en cascade), pour ne pas dire symbolique, et que de nombreux \& accidents» de leur structure hydraulique leur confèrent une tendance fâcheuse à entretenir des oscillations en masse, mettant en jeu de malencontreux vases communiquants, tels que cheminées d'équilibre, reniflards, ou encore les effets d'ondes réfléchies dans des canaux longs et étroits.

Asservir l'ouverture des turbines à la position d'un placide flotteur n'est done souvent qu'une utopie, le flotteur partant en gigues effrénées dès qu'un écart de débit s'est manifesté, mettant en des branles plus ou moins déphasées trois centrales en cascades, deux canaux d'amenée aux ondes voyageuses, et trois chambres d'équilibre plus ou moins en délicatesse avec la fameuse formule de stabilité minimale, dite de Thoma.

Comment donc sortir de ce cycle infernal, au bout duquel le doigté d'un vieux chef d'exploitation sait quelquefois ramener la concorde, grâce à des coups de pouce subtilement nuancés?

En principe, ces systèmes oscillants hydromécaniques sont, grâce aux méthodes modernes, accessibles au calcul. On pourrait donc, à grand renfort de calcul symbolique, mener de savantes études, dont les résultats, artistement présentés, rehausseraient l'éclat de telle grande revue technique.

En pratique, l'application brutale des résultats du calcul conduirait à concevoir, pour chaque cas, un automate particulier, el à rechercher, lors de sa mise en route, les raisons bassement matérielles qui ont faussé les valeurs numériques des optimums calculés : la cheminée d'équilibre n'a pas partout le diamètre porté sur les plans, un canal est malencontreusement étranglé dans un endroit inconnu, ou bien, tout le monde avait oublié un déversoir de sécurité caché dans le recoin d'un ravin sauvage; enfin, qui dira les incertitudes attachées aux caractéristiques d'un régulateur de turbines vétuste, ou volontairement ralenti?

Pour cet ensemble de raisons pratiques, qui donnent au problème son véritable visage, il nous semble préférable de l'aborder d'emblée par son côté pratique. Faisant l'hypothèse que toute centrale au fil de l'eau renferme des « germes» d'instabilité, nous avons imaginé, puis essayé et mis au point un appareillage de réglage comportant des éléments largement réglables de stabilisation. 
Nous pourrons alors, par une simple campagne d'essais de réglage, durant généralement quelques jours au plus, obtenir un réglage suffisamment proche d'un optimum assez peu précis, pour que la centrale réponde aux spécifications requises :

- maintenir la chute au maximum;

- amortir les oscillations, après un changement de régime, dans un temps court (c'està-dire de l'ordre de quelques minutes);

- éviter des oscillations entretenues résiduelles, même de faible amplitude;

- au cours de changements importants de régime, éviter d'atteindre des valeurs dange- reuses de certains paramètres (niveaux maximaux, en particulier);

- obtenir ces réglages sans modification notable aux régulateurs de turbines existants.

Nous allons maintenant décrire sommairement deux régulateurs, destinés principalement anx centrales au fil de l'eau, et conçus pour traiter :

- le premier, les cas simples, ne nécessitant pas de stabilisation particulièrement énergique;

- le second, des cas plus complexes, nécessitant une stabilisation énergique.

\section{II. - MATERIEL STANDARD NEYRPIC POUR L'ASSERVISSEMENT NIVEAU-PUISSANCE DES GROUPES HYDROELECTRIQUES}

Deux types de matériel ont été mis au point pour répondre aux besoins particuliers des asservissements automatiques dans le domaine de l'hydraulique industrielle. Sans restreindre la généralité d'application de ce matériel, nous traiterons l'exemple particulier de l'asservissement de la puissance hydraulique d'un groupe au débit d'apport.

Le premier type d'appareillage réalise, d'une manière très classique, une correspondance de position entre le niveau réglé et l'ouverture de vannage. C'est donc un simple régulateur à «statisme permanent .

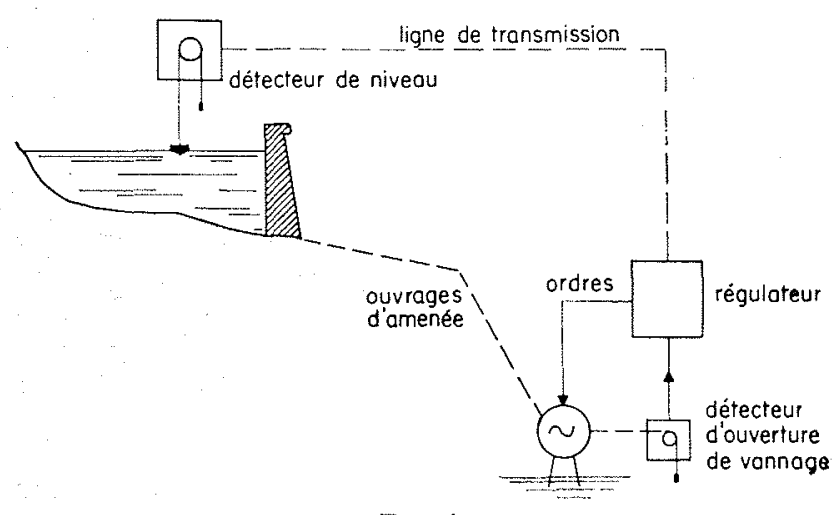

Fig. 1

Schéma général d'un asservissement niveau-puissance d'un groupe hydroèlectrique.
Le deuxième type d'appareillage, plus complexe, introduit dans la boucle de réglage à la fois un statisme permanent et un statisme temporaire; il autorise, de ce fait, des performances intéressantes dans des cas difficiles de régulation.

\section{$1^{\circ}$ Appareillage à statisme permanent, dési- gné par " régulateur à comparateur galva- nométrique ».}

Principe : dans la version la plus simple de l'appareillage, le niveau est détecté par un limnimètre à flotteur à potentiomètre; le potentiomètre du limnimètre est monté en pont avec le potentiomètre d'un « détecteur de déplacement», lié mécaniquement au vannage du groupe. Dans la diagonale du pont, un relais galvanométrique détecte les écarts; par l'intermédiaire d'un ensemble électromécanique, il donne les ordres d'ouverture et de fermeture aul groupe, en agissant, par exemple, sur le moteur du limiteur d'ouverture.

Ce matériel se caractérise avant tout par sa souplesse d'adaptation aux caractéristiques hydrauliques de l'installation. En effet, les deux grandeurs paramétriques: niveau de référence et pente de statisme sont ajustables électriquement à l'aide de simples potentiomètres. 


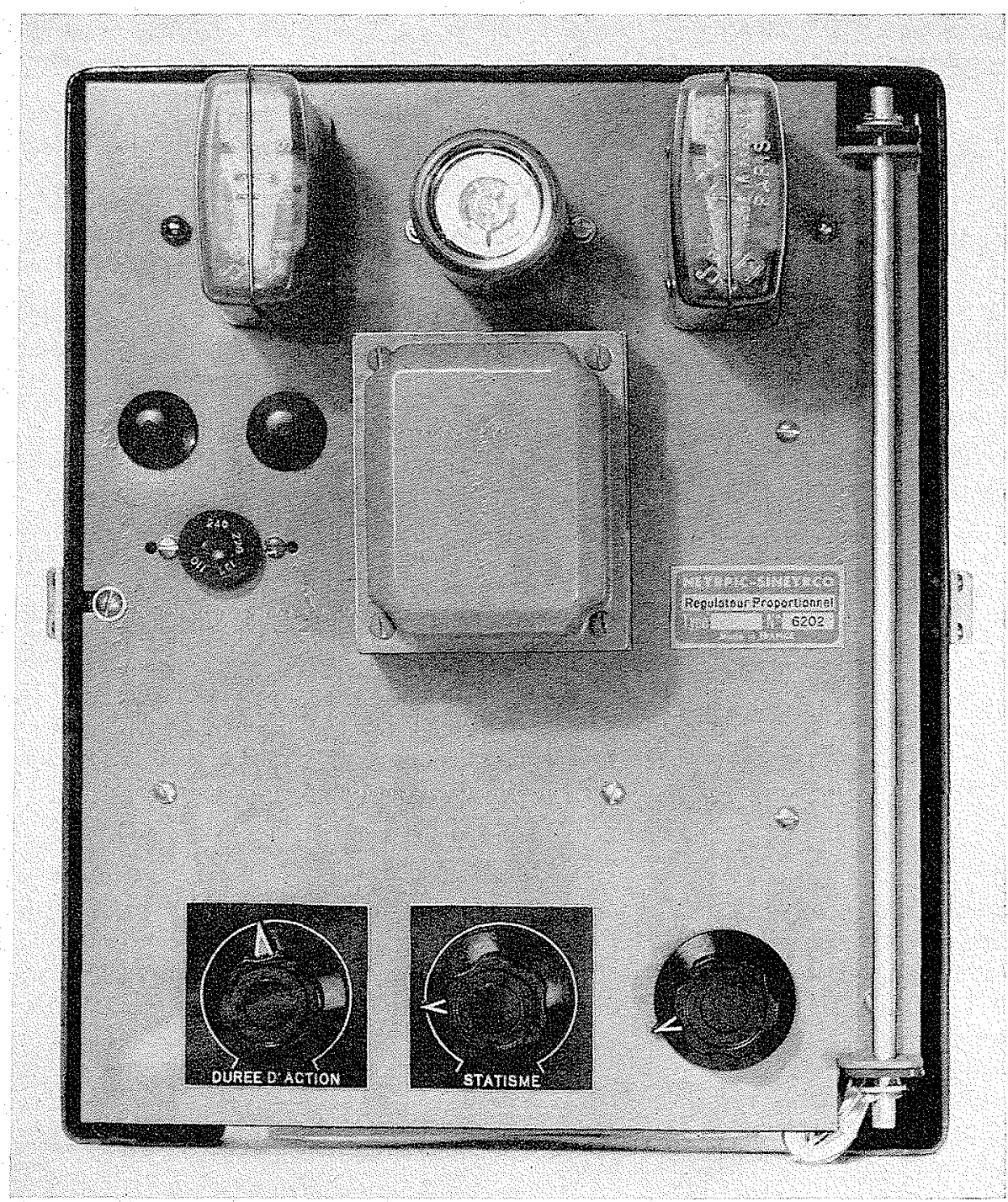

Photo 1

Régulateur à action proportionnelle.

Coffret électrique :

En hant : les denx relais d'ordre et le relais galvanométrique comparateur; En bas : les trois potentiomètres de réglage : durée des ordres, pente de statisme, nivenu de consigne.

Le niveau de référence est ajustable à une valcur quelconque à l'intérieur de la bande de mesure.

L'écart de statisme est ajustable à une valeur comprise entre $10 \%$ et $100 \%$ de la bande de mesure.

DISCONTINUITÉ DES ORDRES : pour remédier à certains défauts, constatés souvent lors de la mise en service d'un asservissement sur une installation déjà ancienne (défauts dûs à des jeux mécaniques dans le régulateur ou dans le vannage), les ordres donnés au groupe sont « décou- pés * en créneaux de durée ajustable par un réglage auxiliaire, et séparés entre eux par un temps constant.

Ce régulateur n'est utilisable qu'avec des servo-moteurs à faible vitesse de manœuvre (ordre de grandeur 30 secondes pour aller de la pleine fermeture à la pleine ouverture).

En résumé : régulateur à statisme permanent, adapté aux grandeurs lentes, faciles à adapter à une installation existante grâce aux réglages électriques de la «grandeur de référence» et de la «pente de statismes. 


\section{$2^{\circ}$ Régulateur à double action.}

Ce régulateur a été mis au point pour la marche automatique an fil de l'eau des groupes hydroélectriques lorsqu'un simple asservissement statique (à statisme permanent) est inutilisable pour des raisons de stabilité.

En général, les installations qui sont justiciables de ce type de régulateur sont celles dans lesquelles un élément introduit un retard de propagation, par inertie ou par propagation d'onde.

L'introduction d'un statisme temporaire dans la boucle d'asservissement, est obtenu d'une manière très classique par un circuit capacitérésistance.

Le détecteur de la grandeur réglée est, comme pour le régulateur précédent, un détecteur potentiométrique; un détecteur de déplacement,

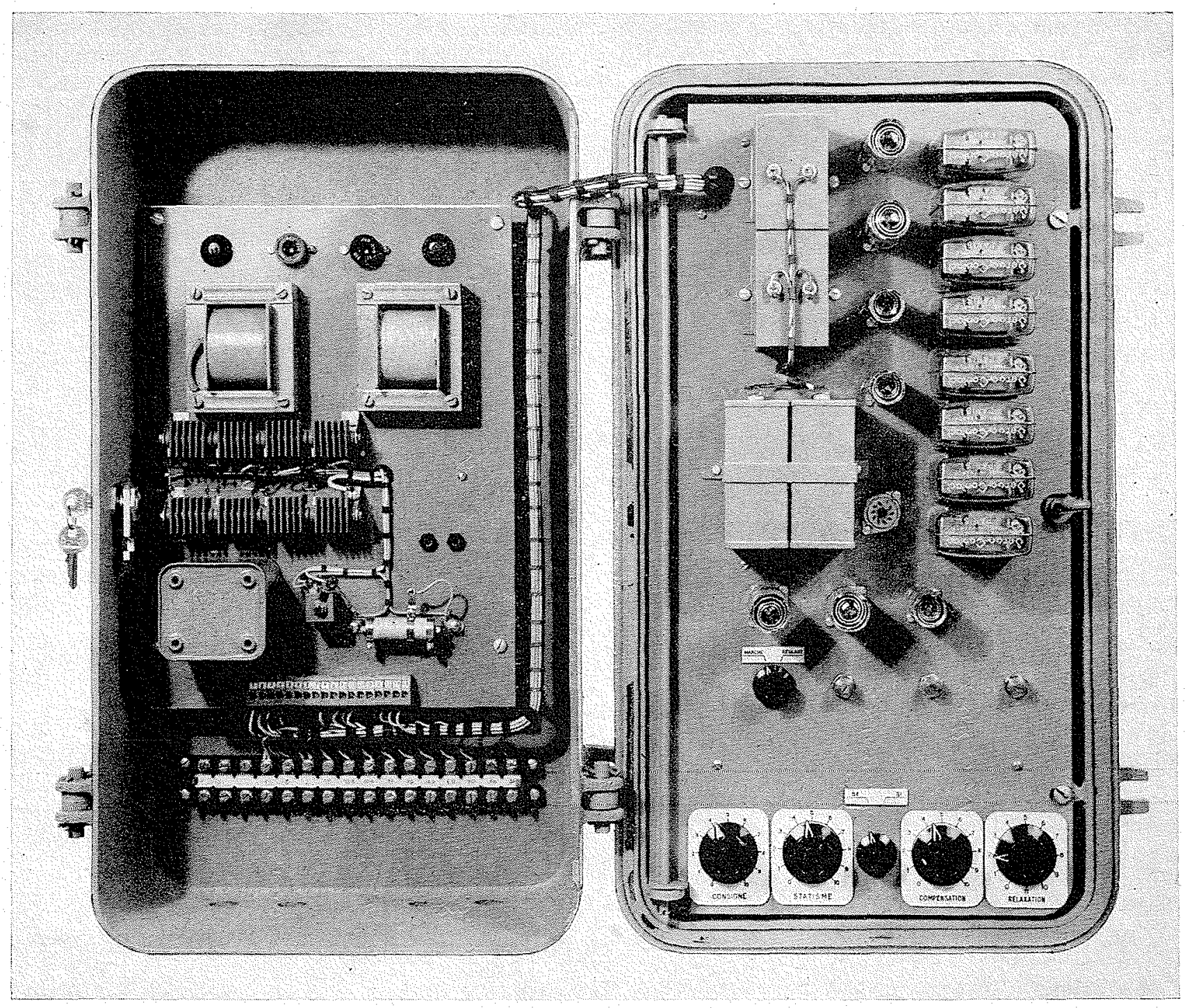

Coffret électronique :

Photo 2

A galache : les circuits d'alimentation et la barrette de raccordement aux organes extérieurs : courant secteur, détecteur de position du vannage, limnimètre à flotteur, moteur de commande du vannage;

A droite : platine électronique avec ses relais d'ordre;

En bas : potentiomètres de réglage de : niveau de consigne, statisme permanent, statisme temporaire (compensation) et temps de relaxation. 
entrainé par le vannage du groupe, introduit dans le régulateur une tension proportionnelle à l'ouverture, tension utilisée à la fois pour les contre-réactions permanente et temporaire.

Le détecteur d'écart est ici, un dispositif électromécanique; les ordres d'ouverture et de fermeture qu'il délivie au groupe sont, comme dans le régulateur précédent, «découpés» dans le temps; mais, dans ce cas, la durée d'ordre est progressive avec l'écart, ce qui est un moyen simple d'obtenir une vitesse de manœuvre grossièrement proportionnelle à l'écart, avec un servo-moteur fonctionnant par tout ou rien.

On retrouve dans ce matériel la souplesse d'adaptation qui caractérise les régulateurs électriques.

Les différents paramètres qui permettent de définir la fonction de transfert la mieux adaptée aux caractéristiques de l'installation sont ajustables par potentiomètres :

- le niveau de référence, à une valeur quelconque à l'intérieur de la bande de mesure;

- l'écart de statisme permanent, entre 0 et $100 \%$ de la bande de mesure;
- l'écart de statisme temporaire, entre 0 et $300 \%$ de la bande de mesure;

- le temps de relaxation, entre 0 et 2500 secondes.

En résumé : régulateur à double action, adapté aux phénomènes présentant des temps caractéristiques importants, facile à adapter grâce aux réglages électriques des différents paramètres.

Pour ces deux appareils, on s'est attaché à obtenir une réalisation particulièrement robuste.

Ces matériels peuvent être complétés par des dispositifs auxiliaires, sans influence sur les performances de la régulation d'une installation, mais qui en facilitent l'exploitation; par exemple : dispositif pour l'équirépartition de la puissance entre deux groupes asservis au niveau; dispositif de commutation des caractéristiques entre heures creuses et heures pleines, etc.

Ces régulateurs peuvent s'asservir à la puissance électrique fournie par le groupe, au lieu de l'ouverture de vannage.

\section{III. - EXEMPLES TYPES D'INSTALLATIONS EQUIPEES DE REGULATEUR NIVEAU-PUISSANCE}

\section{$1^{\circ}$ Installations munies de régulateur à sta- tisme permanent.}

L'installation type pour laquelle la stabilité de l'asservissement niveau-puissance est obtenu par un simple statisme permanent et représentée sur la figure 2.

Le comportement de la cheminée d'équilibre a une grande influence dans la détermination de la pente de statisme. Les installations sans cheminée peuvent travailler avec un statisme très faible.

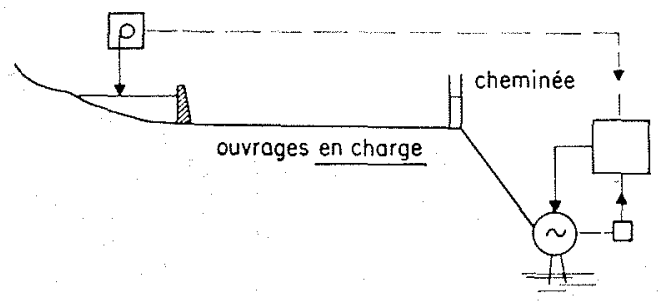

FiG. 2
Installations E.D.F. équipées selon ce schéma dans le département de l'Isère :

-- Pont-Haut,

-- Saint-Maurice,

-. Sassenage,

- Le Rivier, d'Allemont,

- La Bourne.

La liaison électrique entre le détecteur de niveau et le régulateur peut être réalisée soit par une ligne directe à trois conducteurs, soil par un dispositif de télémesure permettant d'utiliser un circuit de caractéristiques quelconques (ligne téléphonique par exemple.)

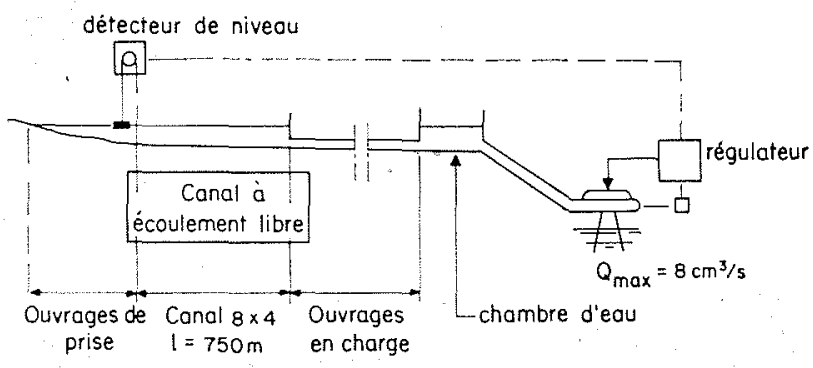

FIG. 3 


\section{$2^{\circ}$ Installations équipées de régulateur à dou- ble action.}

L'utilisation d'un statisme temporaire avec ou sans statisme permanent est motivé dans chaque cas par une raison particulière.

Premier cas : installation impossible à stabiliser avec un statisme permanent seul, l'amplitude de l'écart de niveau nécessaire à la stabilité étant incompatible avec le tracé des ouvrages hydrauliques.

Installation de ce type: Pierre-Eybesse dans la vallée de la Romanche.

DEuxiÈme CAS : ouvrages d'amenée présentant un retard important (canal d'amenée).

Installation de ce type : Pont-de-Claix dans la vallée du Drac.
Le régulateur maintient à une valeur constante de la cote du plan d'eau à la prise; le temps de réponse du canal ( 6 minutes) nécessite l'introduction d'un statisme temporaire dans la boucle d'asservissement.

TroIsı̇̀me cAs : installations pour lesquelles on désire un réglage à niveau constant ou même avec un écart de réglage légèrement négatif.

Dans ces cas, le statisme permanent, soit étant nul, soit agissant dans le sens de l'instabilité, c'est le statisme temporaire seul qui assure la stabilité du réglage.

Installations de ce type : le Verney d'Allemont sur l'Eau d'Olle et La Goule Blanche dans le Vercors.

Cette dernière installation est étudiée en détail par ailleurs.

\section{IV. - UN CAS NON CLASSIQUE D'ASSERVISSEMENT RESOLU PAR UN REGULATEUR STANDARD A DOUBLE ACTION}

L'installation hydroélectrique de La Goule Blanche comprend: retenue de faible surface, galerie en charge, chambre d'eau, conduite forcée.

Pendant les heures creuses, on désire maintenir maximal le stockage dans l'installation, tout en turbinant les apports. Pour que cette condition soit réalisée à tous les débits, il suffit de régler l'ouverture du groupe pour maintenir à une cote constante le niveau dans la prise amont.

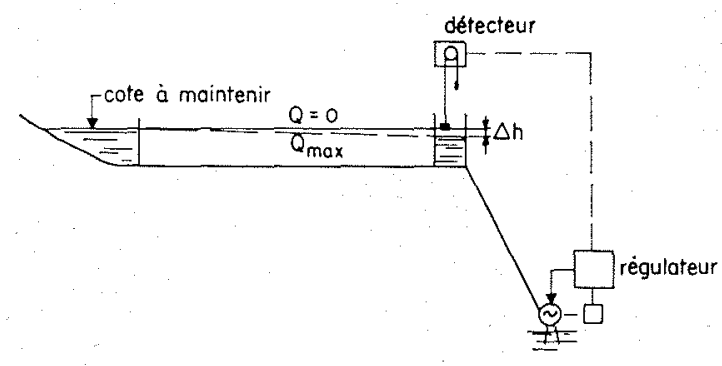

FIg. 4

Pour maintenir le niveau à l'amont, le régulateur doit tenir compte de la perte de charge dans la galerie.
Aucune liaison électrique n'existe entre la prise d'eau et l'usine et, pour éviter l'installation de cette liaison, on a étudié la possibilité d'obtenir une fonctionnement tel que défini cidessus en utilisant le niveau de la chambre de mise en charge comme niveau de réglage. Ceci revient à introduire dans la correspondance entre le niveau (de la chambre d'eau) et l'ouverture $d u$ groupe un statisme inverse (négatif) pour tenir compte de la perte de charge en galerie.

Ce statisme négatif rend l'installation instable par nature.

L'étude a porté sur la possibilité de rendre cette installation stable en utilisant pour l'asservissement niveau-puissance un régulateur à double action :

- une action proportionnelle (statisme permanent) négative qui définit la loi de variation en fonction du débit;

-. une action intégrale (statisme temporaire) dont le rôle est de stabiliser l'installation. 
Moyennant quelques hypothèses simplificatrices, l'équation du mouvement de l'eau dans la chambre d'eau s'établit aisément en partant :

- d'une part, de l'équation qui définit les régimes transitoires dans l'ensemble prise d'eau, galerie, chambre de mise en charge en fonction des fluctuations de débit turbiné;

- d'autre part, de l'équation du régulateur de niveau, c'est-à-dire la relation qui relie les écarts de niveau et les manœuvres du vannage du groupe.

L'équation globale de l'installation est une équation du cinquième ordre, pratiquement inaccessible au calcul classique. Le seul intérêt de son étude est de montrer que, moyennant certaines limitations dans le choix des valeurs des paramètres de réglage (pente de statisme temporaire et temps de relaxation), on peut obtenir que toutes les parties réelles des racines de

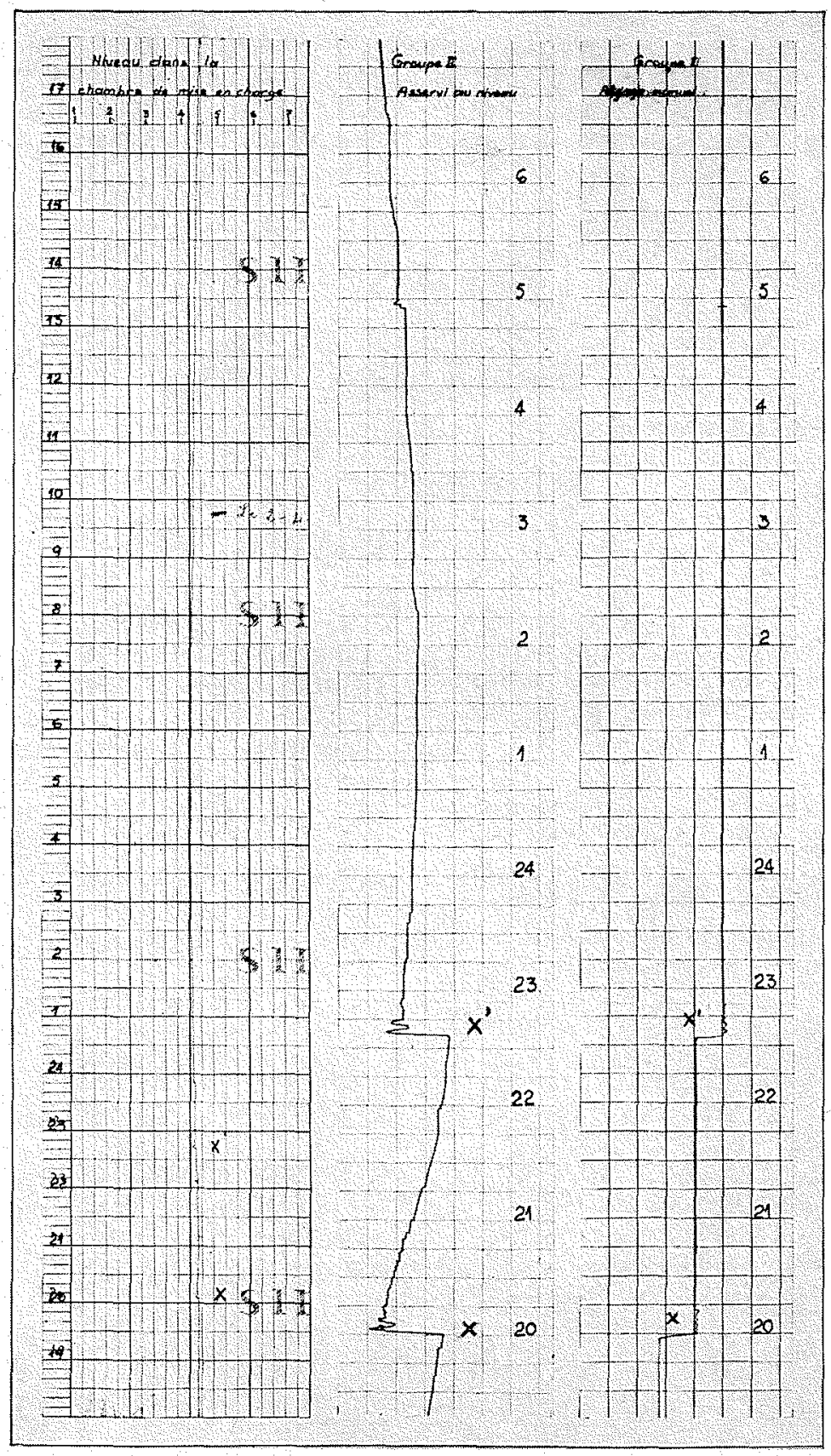

FIG. 5 
l'équation caractéristiques soient négatives, ce qui est le critère de solutions représentant des mouvements amortis, donc d'une installation stable.

Ayant ainsi une justification théorique de la possibilité d'obtenir la stabilité, nous avons compté sur la souplesse d'adaptation du régulateur utilisé pour définir les valeurs optimales à adopter pour les deux paramètres à ajuster (statisme temporaire et temps de relaxation).

Les résultats obtenus sont les suivants :

- le statisme négatif est de 1,60 m (valeur de la perte de charge en galerie au débit maximal).

Après quelques essais, on a obtenu des résultats valables avec les valeurs :

- pente de statisme temporaire : + $3 \mathrm{~m}$;

-- temps de relaxation : $1800 \mathrm{~s}$.

Le graphique (fig. 5) montre un extrait des courbes de niveau et de puissance.

Trois enregistrements simultanés sont reproduits sur ce diagramme :

- à gauche : le niveau dans la chambre de mise en charge;

- au centre: la puissance électrique fournie par le groupe $\mathrm{n}^{\circ} 2$ asservi automatiquement au niveau;

-- à droite: la puissance électrique fournie par le groupe $n^{\circ} 1$, puissance réglable uniquement manuellement.

Pendant la durée d'enregistrement (de $20 \mathrm{~h}$ à $6 \mathrm{~h}$ ) le débit d'apport passe par un maximum (vers $2 \mathrm{~h}$ ); l'opérateur a été amené par deux fois (en $\mathrm{X}$ et $\mathrm{X}^{\prime}$ ) à augmenter la puissance turbinée par le groupe 1 , ceci pour éviter que le groupe 2 ne viemne en butée à sa puissance maximale; ces manouvres manuelles, en créant une variation brutale du débit turbiné, provoquent une perturbation dans l'installation, perturbation qui met un certain temps à s'amortir.

On voit en $\mathrm{X}$ et $\mathrm{X}^{\prime}$ que l'amortissement est acceptable, le groupe reprenant le «fil de l'eau» après un temps de $7 \mathrm{mn}$ environ.

On constate que le niveau dans la chambre de mise en charge baisse au fur et à mesure que le débit turbiné augmente. Le maximum de débit se situe à $2 \mathrm{~h}$, le maximum de niveau vers 2 h $30 \mathrm{mn}$; l'écart est provoqué par le temps de retour du circuit à contre-réaction temporaire.

Puis, le débit turbiné diminue en même temps que le niveau monte. (L'apparente stabilité du niveau entre $3 \mathrm{~h}$ et $7 \mathrm{~h}$ est due d'une part à l'insensibilité au retournement de l'appareil enregistreur; l'appareil régulateur de niveau, qui travaille sur une plage moins importante, est plus sensible aux faibles variations; d'autre part, la réduction d'échelle à la reproduction fait disparaître les petits écarts d'enregistrement).

\begin{abstract}
Run-of-the-river hydro-power plant running costs can be reduced by using simple automatic equipment to control the head on the turbines. The governing problem, which requires the upstream level to be maintained at its maximum height, is frequently complicated by the fact that an automatic control of this type tends to maintain mass oscillations in the system.

A practical approach to this problem is preferable to any attempt at calculating the stabilising elements, for in view of the inaccuracy of the characteristic numerical data for the plant, calculated results are bound to be rather unreliable. This is why electric governing systems in which all the stabilising elements are adjustable within wide limits have been developed. Merely by carrying out a series of simple tests requiring no more than a few days to run, a governor setting can be determined which is sufficiently close to an optimum value to ensure that the plant meets the following specifications:-

a) Maintenance of maximum head.

b) Damping of the oscillations produced by a change of running conditions in a short time (a few minutes).

c) Elimination of even low-amplitude maintained residual oscillations.

d) Avoidance of dangerous values for certain parameters (especially water level) during major changes in ruming conditions.
\end{abstract}


e) The possibility of obtaining these governing conditions without modifying existing turbine governors.

Two types of equipment have been developed to meet the particular needs of automatic control systems in industrial hydraulics, as follow:-

(I) A straightforward 'permanent speed droop' governor based on the conventional principle of linking water level regulation with gate settings. The values of the two parameters -reference level and speed droop curve-are varied by means of simple potentiometers and the entire system is electro-mechanical.

(II) A more complex type of governing system in which both permanent and temporary speed droop are set up in the feedback circuit, thus allowing very good results to be achieved in cases where regulation in difficult.

Installations which merit the use of this type of governor are usually those in which one element causes control lag either by inertia or wave propagation.

Temporary speed droop is set up in the feedback circuit by means of a conventional capacity-resistance circuit.

The various parameters used to define the best transfer function for the characteristics of the installation are adjustable by potentiometer, as follow:-

(I) The reference level, which can be set to any value within the range of measurement.

(II) Permanent speed droop variation, between 0 and $100 \%$ of the range of measurement.

(III) Temporary speed droop variation, between 0 and $300 \%$ of the range of measurement.

(IV) Damping time of between 0 and 2,500 seconds. 